\title{
Determinants of mastitis in women in the CASTLE study: a cohort study
}

\author{
Meabh Cullinane ${ }^{1}$, Lisa H. Amir ${ }^{\text {** }}$, Susan M. Donath ${ }^{2,3}$, Suzanne M. Garland ${ }^{2,4,5}$, Sepehr N. Tabrizi²,4, \\ Matthew S. Payne ${ }^{6}$ and Catherine M. Bennett ${ }^{7}$
}

\begin{abstract}
Background: Mastitis is an acute, debilitating condition that occurs in approximately $20 \%$ of breastfeeding women who experience a red, painful breast with fever. This paper describes the factors correlated with mastitis and investigates the presence of Staphylococcus aureus in women who participated in the CASTLE (Candida and Staphylococcus Transmission: Longitudinal Evaluation) study. The CASTLE study was a prospective cohort study which recruited nulliparous women in late pregnancy in two maternity hospitals in Melbourne, Australia in 2009-2011.

Methods: Women completed questionnaires at recruitment and six time-points in the first eight weeks postpartum. Postpartum questionnaires asked about incidences of mastitis, nipple damage, milk supply, expressing practices and breastfeeding problems. Nasal and nipple swabs were collected from mothers and babies, as well as breast milk samples. All samples were cultured for S. aureus. "Time at risk" of mastitis was defined as days between birth and first occurrence of mastitis (for women who developed mastitis) and days between birth and the last study time-point (for women who did not develop mastitis). Risk factors for incidence of mastitis occurring during the time at risk (Incident Rate Ratios [IRR]) were investigated using a discrete version of the multivariable proportional hazards regression model.
\end{abstract}

Results: Twenty percent (70/346) of participants developed mastitis. Women had an increased risk of developing mastitis if they reported nipple damage (IRR 2.17, $95 \% \mathrm{Cl} 1.21,3.91$ ), over-supply of breast milk (IRR 2.60, $95 \% \mathrm{Cl}$ 1.58, 4.29), nipple shield use (IRR $2.93,95 \% \mathrm{Cl} 1.72,5.01)$ or expressing several times a day (IRR $1.64,95 \% \mathrm{Cl} 1.01,2.68)$. The presence of $S$. aureus on the nipple (IRR 1.72, $95 \% \mathrm{Cl} 1.04,2.85)$ or in milk (IRR 1.78, $95 \% \mathrm{Cl} 1.08,2.92)$ also increased the risk of developing mastitis.

Conclusions: Nipple damage, over-supply of breast milk, use of nipple shields and the presence of S. aureus on the nipple or in breast milk increased the mastitis risk in our prospective cohort study sample. Reducing nipple damage may help reduce maternal breast infections.

Keywords: Breastfeeding, Mastitis, Breast infection, Staphylococcus aureus

\section{Background}

Mastitis is an acute, debilitating condition that occurs in approximately $20 \%$ of breastfeeding women who experience a red, painful breast with fever [1-3]. It is a distressing condition which negatively impacts the daily activities of sufferers [4, 5]. Most episodes of mastitis occur in the first two months postpartum [1-3] and recurrence rates of up to about $10 \%$ have been reported

\footnotetext{
* Correspondence: l.amir@latrobe.edu.au

'Judith Lumley Centre (formerly Mother \& Child Health Research), La Trobe

University, Melbourne, VIC 3000, Australia

Full list of author information is available at the end of the article
}

$[2,3,6]$. Early management of mastitis involves general measures to improve drainage of the breast and reduce inflammation [7]. Medical management by general practitioners in the community usually involves oral antibiotics [8], with minimal interference to breastfeeding, however some women require hospitalisation or develop a breast abscess [9]. An earlier Melbourne-based study found approximately $3 \%$ of mastitis sufferers developed a breast abscess subsequent to a mastitis episode [10].

Nipple pain and damage, maternal stress and fatigue, attachment difficulties, milk stasis and restrictions from a tight fitting bra have been implicated as contributing 
to mastitis $[1,3,11-14]$. Whereas some studies have found that women with mastitis are not more or less likely to continue breastfeeding than other women [1], others have found that mastitis sufferers are more likely to breastfeed for longer than women without mastitis $[15,16]$. There have been reports that women receiving private or birth centre care are also more likely to develop mastitis than other women $[1,17,18]$. Anecdotally breast milk expression has been linked to mastitis, and expressing breast milk has become more prevalent, even for healthy term infants $[19,20]$, however this has not been fully investigated. A recent study using a case-control design found an association between using breast pumps and mastitis, but questionnaire data were collected retrospectively, not at the time of mastitis, so it is unclear if the expressing was a risk for or a consequence of mastitis [21].

Traditionally, Staphylococcus aureus has been considered the most common aetiological agent of mastitis and is frequently isolated in cases of infective mastitis and breast abscesses [22, 23]. In a case-control study, Amir et al. found that women with mastitis were more likely to have $S$. aureus present in breast milk than women in the control group [24]. At present, the role of other organisms such as coagulase-negative S. aureus is unclear $[25,26]$.

The aims of this prospective study are to describe the incidence and correlates of mastitis in breastfeeding women within the first two months after birth. We also investigated the presence of $S$. aureus in milk during mastitis episodes compared to milk samples collected from healthy women during the study.

\section{Methods}

\section{Sample}

The CASTLE (Candida and Staphylococcus Transmission: Longitudinal Evaluation) study investigated the microorganisms involved in the development of mastitis and "breast thrush" among breastfeeding women [27], and confirmed the role of Candida species in the symptoms of "breast thrush" [28]. Three hundred and sixty nulliparous women were recruited from two hospitals in Melbourne, Australia: the Royal Women's Hospital, (RWH) a public tertiary referral centre, and Frances Perry House (FPH), a private co-located hospital. At recruitment participants completed a questionnaire asking about maternal age, gestational age, intended length of breastfeeding duration, highest level of educational attainment, marital status and previous staphylococcal infections. Following birth, participants were followed-up six times: in hospital and then at home weekly until four weeks postpartum and at eight weeks by telephone.

Eligibility criteria for the study were: between $18-50$ years of age; nulliparity; $\geq 36$ weeks pregnant at recruitment; singleton pregnancy; breastfeeding intention for at least eight weeks postpartum; sufficient proficiency in English to complete written questionnaires and a telephone interview; residing $\leq 16 \mathrm{~km}$ from Melbourne Central Business District (CBD). Criteria for exclusion were: medical conditions which do not allow breastfeeding; breast reduction surgery; dermatitis on nipple during pregnancy; under care of the Women's Alcohol and Drug Service (WADS); under care of Mental Health Service or social worker.

\section{Data measures \\ Questionnaires}

Self-administered questionnaires completed in hospital after birth and at weeks $1,2,3,4$ and 8 postpartum collected information about milk supply, nipple damage and mastitis. At weeks 1, 2, 3, 4 and 8 postpartum women were asked to rate their milk supply since their last interview. They could state that they were "producing enough milk for their baby", "not producing enough milk", "over-producing breast milk" or that their "milk supply varied". They were also asked: "In the last week, have you been expressing breast milk?" The women who had expressed in the last week were asked "on average, how often do you express?" and could select one of the following options: "once this week", "several times this week", "once a day"; "several times a day" or "it varies" [29].

At all time-points postpartum, participants were asked whether they had any nipple damage. If they had, they could define this damage as a small graze/crack $(<2 \mathrm{~mm}$ in length), a moderate graze/crack (2 to $9 \mathrm{~mm}$ in length) or a severe graze/crack ( $\geq 10 \mathrm{~mm} \& /$ or yellow colour present).

Participants were given the opportunity to report any problems they had with breastfeeding. At weeks 1, 2, 3, 4 and 8 postpartum women were asked whether they had any problems with breastfeeding since their last interview. They were supplied with a list of problems, including attachment issues, over or under-supply of breast milk, use of nipple shield to feed, having an unsettled infant, or an infant not interested in feeding. Women were asked about nipple pain and whether they had used any creams or ointments on their nipples, including use of hydrated polymer (hydrogel) dressings (results have been published separately) [30,31]. They could also state a breastfeeding problem which was not listed.

There is no standard way of diagnosing mastitis. Therefore, we asked about a range of breast symptoms and associated fever or flu-like symptoms, as has been used previously $[1,32]$. For this analysis the definition of mastitis was the development of at least two breast symptoms (pain, redness, lump) and at least one systemic symptom (fever or flu-like symptoms) [1,32] or treatment of mastitis with antibiotics. Women were asked to rate how mastitis had interfered with breastfeeding and with 
activities of daily living on a scale of 0 to 4 where 0 was "no interference" and 4 was "quite a bit of interference" [5].

If a participant ceased breastfeeding during the first four weeks postpartum, the subsequent home visits were not conducted but the participant was contacted at eight weeks to complete the final questionnaire by telephone.

\section{Microbiology}

Women provided nasal and nipple (both nipples) swabs and breast milk samples (both breasts). Nasal and oral swabs were obtained from infants. In addition, all women were given a $70 \mathrm{ml}$ sterile container (Sarstedt Australia Pty. Ltd.) and asked to collect a sample of milk from the affected breast should they develop mastitis during the first eight weeks postpartum between their scheduled weekly visits. Samples were collected and cultivated for S. aureus as described in the study protocol [27].

\section{Data analysis}

Descriptive analysis included maternal demographic characteristics and details about the birth, breastfeeding duration, breastfeeding difficulties, and episodes of mastitis. Using information from the successive postpartum time-points, we investigated possible predictors of (or risk factors for) mastitis incidence. We defined "time at risk" of mastitis as days between birth and first occurrence of mastitis (for women who developed mastitis) and days between birth and the last study time-point (for women who did not develop mastitis). We used a discrete version of the multivariate proportional hazards regression model [33] to investigate whether mothers' reported nipple damage, "over-production of milk", breastfeeding attachment problems, use of nipple shield to feed, milk expressing, use of hydrogel dressings, and presence of $S$. aureus in samples collected from swabs and breast milk samples were risk factors for mastitis incidence if they occurred during the time at risk. For each of the risk factors investigated (mothers' reported nipple damage, "over-production of milk", etc), we estimated an incidence rate ratio: the incidence rate of mastitis in those WITH the risk factor during the "time at risk" divided by the incidence rate of mastitis in those WITHOUT the risk factor during the "time at risk". We used the glm command in Stata with link function $=$ cloglog, family = binomial to estimate the rate ratios. This method uses time at risk and time-varying risk factors. Comparisons are presented using incident rate ratios, $95 \%$ confidence intervals (CIs), and $p$-values. All analyses were carried out using Stata 13 software [34].

\section{Ethics approval}

The study received approval from La Trobe University Human Ethics Committee (LTU UHEC No. 06-078), the RWH Human Research Ethics Committee (RWH HREC
No. 06-41) and the Medical Advisory Committee at FPH. All participants provided written informed consent.

\section{Results \\ Sample}

Three hundred and sixty women were recruited to the CASTLE study between October 2009 and May 2011. During the study, 14 women withdrew after giving birth, leaving 346 women available for data collection (survey results and microbiological data) at defined time-points postpartum.

Information about the main socio-demographic characteristics of the sample is shown in Table 1. Overall, the women's mean age was 32.7, with a range of between 19 and 44 years. Seventy seven percent of participants $(n=267)$ held a University degree or higher, indicating that this cohort represents a highly educated sample of the general population. Breastfeeding intention ranged from one month to 24 months, with a mean of ten months. The method of birth was Caesarean section for $45 \%$ of study participants. Ten percent of participants $(n=36)$ reported suffering from previous staphylococcal infections, such as boils, abscesses or sores inside the nose.

\section{Incidence of mastitis}

Seventy women experienced mastitis, representing $20 \%$ of participants (70/346). There was no difference in the prevalence of mastitis among public and private patients; (40/192 private patients, 30/124 public patients experienced mastitis). There were 97 episodes of mastitis in total. Figure 1 shows the proportion (\%) of mastitis

Table 1 Characteristics of CASTLE study participants

\begin{tabular}{|c|c|c|}
\hline Maternal characteristics $(n=346)$ & $n$ & Percent \\
\hline \multicolumn{3}{|l|}{ Hospital } \\
\hline Royal Women's Hospital (public) & 154 & 44.5 \\
\hline Frances Perry House (private) & 192 & 55.5 \\
\hline Age (years - mean, SD, range) & $\begin{array}{l}32.7 \\
(4.1,19-44)\end{array}$ & \\
\hline \multicolumn{3}{|l|}{ Education level } \\
\hline Tertiary degree or higher & 267 & 77 \\
\hline Other & 79 & 23 \\
\hline $\begin{array}{l}\text { Breastfeeding intention } \\
\text { (months - mean, range) }\end{array}$ & $9.7(1-24)$ & \\
\hline \multicolumn{3}{|l|}{$\begin{array}{l}\text { Previous staphylococcal infections } \\
\text { (e.g. boils, abscesses, sores inside the nose) }\end{array}$} \\
\hline Yes & 284 & 82.1 \\
\hline No & 36 & 10.4 \\
\hline Unsure & 26 & 7.5 \\
\hline Caesarean birth & 156 & 45 \\
\hline
\end{tabular}




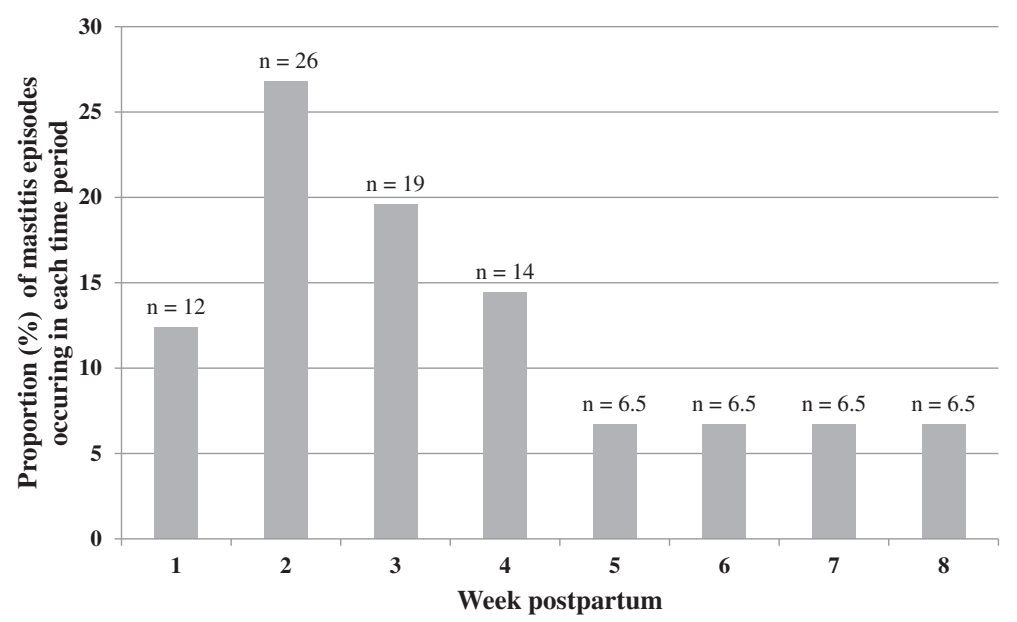

Fig. 1 Proportion (\%) of mastitis episodes per week in women in the CASTLE study ( $n=97$ mastitis episodes in 70 women). Mastitis episodes in weeks 5 to 8 were averaged; 24 women experienced mastitis in this time period

episodes per week. Because women were contacted at eight weeks postpartum and asked about mastitis episodes they experienced since their last home visit at week 4, mastitis episodes experienced by women in weeks 5 to 8 were averaged. Twenty four women experienced mastitis in this time period. The majority of mastitis episodes (71 episodes, $73 \%$ of the total) occurred in the first four weeks postpartum. Forty eight women experienced mastitis once, while 17 and 5 women experienced this condition two and three times respectively.

Mastitis sufferers were asked to rate how mastitis had interfered with both breastfeeding and with activities of daily living on a scale of 0 (no interference) to 4 (quite a bit of interference). Fifty percent (35/70) of women with mastitis reported that it interfered with breastfeeding (scored 3 or 4 on a 4-point scale) and $60 \%(42 / 70)$ of women with mastitis reported that it interfered with activities of daily living (scale as above).

\section{Correlates of mastitis}

The total "time at risk" (i.e. from birth to first mastitis or last interview postpartum if no mastitis) was fifty personyears. Potential correlates of mastitis incidence were investigated using information obtained from women at each time-point on (i) their expressing habits, (ii) self-reported breastfeeding problems and (iii) whether $S$. aureus was isolated at each time-point in nipple and milk samples from women or oral and nasal samples from infants (Table 2). Specific potential determinants of mastitis investigated were: nipple damage; expressing several times per day; attachment problems; over-supply of breast milk; use of nipple shields; use of hydrogel dressings; S.aureus cultured from nipple swabs collected from hospital onwards; S. aureus cultured from milk samples from hospital onwards and S. aureus cultured from the infant (either from oral or nasal swabs) from hospital onwards.

At each time-point in the first 4 weeks postpartum, women who had already reported any nipple damage had a more than two-fold increased risk of developing mastitis compared to women without nipple damage (Incident Rate Ratio (IRR) 2.17, 95 \% CI 1.21, 3.91). Similarly, women reporting an over-supply of breast milk, attachment problems, nipple shield use or who expressed several times a day had a significantly increased risk of developing mastitis compared to women who did not report these (Table 2). However, women who reported any expressing in the week(s) before questionnaire administration did not have an increased risk of developing mastitis (IRR 1.35, $95 \%$ CI $0.70,2.57)$. Mothers with $S$. aureus isolated from their nipple and/or breast milk had an increased risk of subsequently developing mastitis (nipple IRR 1.72, 95 \% CI 1.04, 2.85, milk IRR 1.78, 95 \% CI 1.08, 2.92). The proportion of women who had S. aureus isolated in their milk (Fig. 2) or nipple or milk (Fig. 3), are shown at weeks 1, 2, 3 and 4. Women who developed mastitis in the first 4 weeks were more likely to have $S$. aureus isolated at each visit after week 1 . There was some evidence to suggest that use of hydrogel dressings or S. aureus isolation from either infant nasal or oral swabs led to an increased risk of developing mastitis (hydrogel dressing use: IRR $=1.7 ; 95 \% \mathrm{CI}$ 0.96, 3.09; S. aureus isolation from either infant nasal or oral swabs IRR 1.74, $95 \%$ CI 0.97, 3.11). However, maternal nasal carriage of $S$. aureus did not increase the risk of risk of developing mastitis (IRR 1.11, $95 \%$ CI $0.68,1.81$ ). 
Table 2 Correlates of mastitis incidence in women in the CASTLE study ( $\mathrm{n}=346 ; 70$ women with mastitis)

\begin{tabular}{|c|c|c|c|c|c|c|}
\hline $\begin{array}{l}\text { Correlate } \\
\text { (occurring from hospital onwarc }\end{array}$ & & $\begin{array}{l}\text { Number of women with at } \\
\text { least one mastitis event }\end{array}$ & $\begin{array}{l}\text { Person-years at risk of } \\
\text { first mastitis event }\end{array}$ & $\begin{array}{l}\text { Estimated Incidence } \\
\text { Rate Ratio (IRR) }\end{array}$ & $95 \%$ Cl for IRR & $p$ value \\
\hline Any nipple damage & yes & 55 & 31.8 & 2.17 & $1.21,3.91$ & 0.01 \\
\hline & no & 15 & 18.3 & & & \\
\hline "Over-producing milk" & yes & 26 & 9.7 & 2.60 & $1.58,4.29$ & $<0.005$ \\
\hline & no & 44 & 40.4 & & & \\
\hline Attachment problem & yes & 45 & 24.3 & 1.96 & $1.18,3.24$ & 0.009 \\
\hline & no & 25 & 25.8 & & & \\
\hline Use of nipple shield & yes & 23 & 7.4 & 2.93 & $1.72,5.01$ & $<0.005$ \\
\hline & no & 47 & 42.7 & & & \\
\hline Expressing several times a day & yes & 33 & 17.7 & 1.64 & $1.01,2.68$ & 0.047 \\
\hline & no & 37 & 32.4 & & & \\
\hline Use of hydrogel dressings & yes & 15 & 7.0 & 1.72 & $0.96,3.09$ & 0.071 \\
\hline & no & 55 & 43.0 & & & \\
\hline S. aureus isolated from nipple & yes & 46 & 26.5 & 1.72 & $1.04,2.85$ & 0.035 \\
\hline & no & 24 & 23.5 & & & \\
\hline S. aureus isolated from milk & yes & 44 & 24.6 & 1.78 & $1.08,2.92$ & 0.023 \\
\hline & no & 26 & 25.4 & & & \\
\hline S. aureus isolated from infants & yes & 55 & 34.1 & 1.74 & $0.97,3.11$ & 0.062 \\
\hline & no & 15 & 15.9 & & & \\
\hline
\end{tabular}

\section{S. aureus in breast milk samples}

Breast milk samples were collected from 20 women who reported mastitis at the time of collection or the day before milk collection. Because mastitis could be present in one breast or both breasts, in total 27 mastitis milk samples were collected from these 20 women (Table 3). Fifty nine percent (16/27) of these milk samples were culture-positive for $S$. aureus (95\% CI $39 \%, 78 \%)$.

Breast milk samples from women who did not report mastitis at weeks $1,2,3$ and 4 postpartum were also cultivated for S. aureus (Table 3). Data are presented as S. aureus positive milk samples collected per week. Thirty two percent $(207 / 657)$ of the milk samples collected at week 1 were culture positive for $S$. aureus. Culture positive milk samples at weeks 2,3 and 4 were $26 \%(172 / 660), 18 \%(117 / 648)$ and $15 \%(94 / 642)$ respectively. $S$. aureus was isolated more frequently in milk samples collected from women who reported mastitis at the time, or the day before milk collection, than from milk samples collected when women did not report mastitis (Table 3 ).
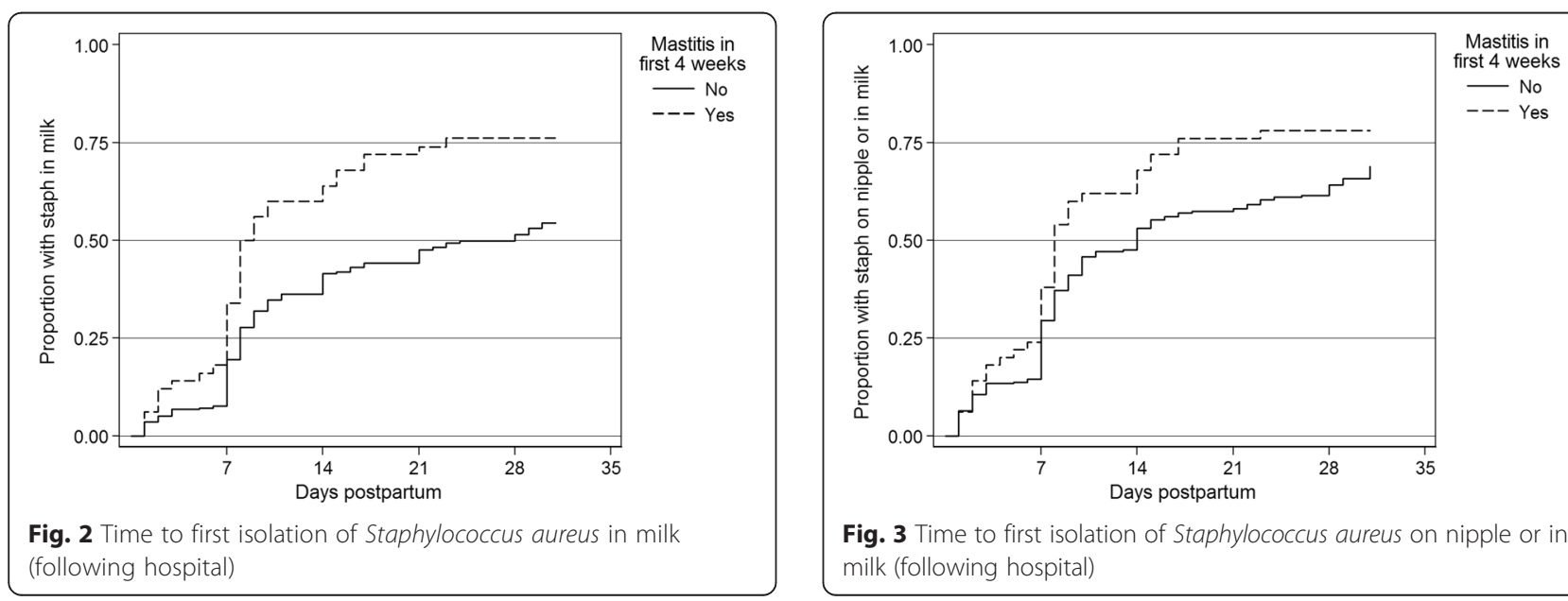

Fig. 3 Time to first isolation of Staphylococcus aureus on nipple or in milk (following hospital) 
Table 3 Milk samples collected in women with and without symptoms of mastitis in which $\mathrm{S}$. aureus was isolated (total number of women $=346$ )

\begin{tabular}{lll}
\hline S. aureus-positive milk samples & \\
\hline Women with mastitis $(n=27)^{\mathrm{b}}$ & 16 & $\%(95 \% \mathrm{Cl})$ \\
Women without mastitis: & & $59.3(39,78)$ \\
Week $1(n=657)$ & 207 & $31.5(28,35)$ \\
Week 2 $(n=660)$ & 172 & $26.1(23,29)$ \\
Week $3(n=648)$ & 117 & $18.1(15,21)$ \\
Week $4(n=642)$ & 94 & $14.6(12,17)$ \\
\hline
\end{tabular}

${ }^{\mathrm{a}}$ Samples were collected separately for each breast

${ }^{\mathrm{b}}$ Milk collected from women who reported mastitis at the time of collection or the day before milk collection

\section{Discussion}

\section{Main findings}

During the eight week study period, $20 \%$ of participants developed mastitis; the majority of episodes were reported in the first four weeks postpartum. Women had an increased risk of developing mastitis if they reported nipple damage, problems with attachment, an oversupply of breast milk, nipple shield use or if they reported expressing breast milk several times a day. The presence of $S$. aureus on the nipple or in breast milk samples also increased the risk of developing mastitis using a prospective study design.

\section{Strengths and limitations}

A strength of this study is that participants had not previously breastfed or experienced mastitis; they were recruited prior to starting breastfeeding. Some studies have suggested that women who have previously experienced mastitis are more likely to experience this condition again with subsequent infants [13, 14, 35]. Our cohort was followed until eight weeks postpartum with milk samples, breast and nipple analysis and detailed questionnaires collected concurrently at defined timepoints, facilitating a comprehensive investigation of the development of mastitis in the early weeks postpartum. We also collected oral and nasal samples from infants to investigate whether infant colonisation with $S$. aureus could represent a potential route of transmission contributing to the development of mastitis. The prospective data collection enabled us to conduct a novel time-to-event analysis, whereas most studies of mastitis have used retrospective data collection [21, 24].

A limitation of this study is that we relied on maternal self-report for mastitis. We tried to overcome this by using a strict definition of mastitis; two breast symptoms and one systemic symptom, or treatment of mastitis with antibiotics. We also relied on self-report for problems with breastfeeding such as an over-supply of breast milk, problems with attachment and nipple shield use. Further, although we followed the cohort until eight weeks postpartum, we have microbiological data only to four weeks postpartum and so we were unable to carry out microbial assessments after this time.

\section{Interpretation}

We used a strict definition of mastitis in this study to estimate the proportion of breastfeeding women who experienced a clinically significant illness. To reduce bias, we did not ask participants about mastitis directly. Using our definition, $20 \%$ of the study population experienced at least one episode of mastitis, which is in agreement with several other Australian studies [1, 2, 36, 37]. However, in contrast to other studies $[1,17]$, mastitis was not more prevalent among private patients than women receiving public hospital care. This may be due to our small sample size or the fact that women in our study were followed only until eight weeks postpartum unlike previous studies where participants were followed-up at three months [17] and six months [1] after birth. It has been shown that most episodes of mastitis occur in the early weeks postpartum $[1,2]$. In accordance with this, approximately three-quarters of the mastitis episodes occurred in the first four weeks postpartum in this study. Episodes of mastitis peaked at two weeks postpartum and decreased steadily after this point.

We investigated factors that could be correlated with the development of mastitis. We confirmed the important role of nipple damage as well as problems with attachment, over-supply of breast milk and use of a nipple shield in the development of mastitis. Since over $90 \%$ of participants used nipple creams we did not include this in our analysis [31]. It is clear that damage to the nipple can contribute to mastitis [1-3, 12, 38]. Nipple damage occurs most frequently in the early postpartum period, suggesting that trauma to the nipple provides a portal of entry for microorganisms from the damaged skin into the nipple, leading to the development of mastitis $[1,3,38]$. Preventing nipple trauma and improving the management of damaged nipples could reduce the risk of developing mastitis among breastfeeding women. We confirmed previous reports that attachment problems are also associated with the development of mastitis [12]. It may be that incorrect attachment leads to nipple trauma which then contributes to the development of mastitis. Further, the fact that nipple shield use was associated with mastitis in our population suggests that some women who had nipple trauma used nipple shields to feed.

Participants who indicated that they had an oversupply of breast milk were also more likely to develop mastitis. We postulate that mastitis is more likely to occur in women with an over-supply of breast milk as 
they may experience more engorgement and milk stasis, which are known contributors to the development of mastitis $[12,14]$. This study adds to the evidence that expressing several times each day is correlated with mastitis. Women who reported any expressing were not more likely to experience mastitis, however women who reported expressing several times a day had a higher risk of developing mastitis. Women may be using regular expressing to manage nipple trauma or an over-supply of milk. Possibly, the increased practice of regular expressing $[20,39]$, may be exacerbating over-supply and the risk of mastitis in some cases.

This study also investigated the role of S. aureus in the development of mastitis. Although nasal carriage of $S$. aureus plays a role in some infections [40], this study, as well as the previous study by Amir et al. found no association between maternal nasal carriage of this bacteria and mastitis [24]. Women who were culture-positive for $S$. aureus on their nipples or in their expressed breast milk also had an increased risk of developing mastitis. As S. aureus is the usual pathogen associated with mastitis in breastfeeding women [22-24] we also investigated the presence of S. aureus in breast milk from women with and without mastitis. Milk samples collected from women who reported mastitis at the time of, or the day prior to milk collection were more likely to grow $S$. aureus compared to samples from women without mastitis. This is in agreement with previous reports [22-24], and highlights the role of this species in the development of mastitis. Interestingly, many milk samples from healthy women were also positive for $S$. aureus, confirming the presence of this organism as a commensal on the skin and in the milk of healthy women who did not develop mastitis [41]. As Ingman et al. explained: "the interactions between inflammatory stimuli, including pathogenic bacteria, and other components of the microbiome, as well as the host immune response, are all likely to contribute to shaping the severity of mastitis, duration of symptoms and resolution of the disease" ([42] p. 1).

It is clear that mastitis impacts negatively on women's lives; participants reported that their mastitis symptoms interfered with breastfeeding and with activities of daily living. In accordance with a previous study [5], participants reported that their symptoms had a greater impact on activities of daily living than on breastfeeding. The fact that mastitis has such a negative influence on women's lives underlines the need to investigate factors that could be varied to reduce or prevent mastitis. The prevention or early treatment of damaged nipples, active management of breast engorgement and early intervention to help with positioning and attachment may potentially reduce the risk of mastitis in breastfeeding women.

\section{Conclusion}

Twenty percent of CASTLE study participants developed mastitis in the first eight weeks postpartum and this had a negative impact on their lives. Nipple damage, problems with attachment, over-supply of breast milk, expressing several times a day, use of a nipple shield and S. aureus presence on the nipple or in expressed breast milk increased the risk of developing mastitis in our study sample. Education on optimal positioning and attachment leading to a reduction in nipple damage may reduce the incidence of mastitis among breastfeeding women [43].

\section{Competing interests}

The authors declare that they have no competing interests.

\section{Authors' contributions}

LHA conceived the study. All authors have contributed to the development of the study. SMD managed the data and conducted the statistical analyses. SMG, SNT, CMB and MSP provided microbiological expertise into study design and analysis. MC was the project coordinator and contributed to the questionnaire design. MSP was the research scientist. MC drafted the manuscript. All authors read and approved the final manuscript.

\section{Authors' information}

MSP contributed to this publication while at the Judith Lumley Centre, La Trobe University and University of Melbourne Department of Obstetrics and Gynaecology, The Royal Women's Hospital.

\section{Acknowledgements}

We gratefully acknowledge the CASTLE study participants and women who assisted by piloting the initial documentation; staff at the Royal Women's Hospital and Frances Perry House; members of the CASTLE study research team involved in sample collection and analysis (Wendy Thornton, Eve Urban, Jenny Halliday, Ruth Berkowitz, Lulusha Jayawardena, Miranda Buck, Carmel Jeffers, and Rhys Staley); and members of the CASTLE Reference Group (Belinda Barnes, Wendy Brodribb, Amanda Cooklin, Jane Fisher, Liz McGuire, Kate Mortensen, Anita Moorhead, Ross Pagano, Marie Pirotta, Christine Scott, and Narelle Warren).

The major funding was provided by the National Health and Medical Research Council Project Grant (541907) in 2009-2010. Additional funding was received by the Faculty of Health Sciences, La Trobe University (2011-2012), and from the Helen Macpherson Smith Trust (2011-2012).

\section{Author details}

Judith Lumley Centre (formerly Mother \& Child Health Research), La Trobe University, Melbourne, VIC 3000, Australia. ${ }^{2}$ Murdoch Childrens Research Institute, The Royal Children's Hospital, Parkville, VIC 3052, Australia. ${ }^{3}$ University of Melbourne Department of Paediatrics, The Royal Children's Hospital, Parkville, VIC 3052, Australia. “Women's Centre for Infectious Diseases, Royal Women's Hospital, Parkville, VIC 3052, Australia. ${ }^{5}$ University of Melbourne Department of Obstetrics and Gynaecology, The Royal Women's Hospital, Parkville, VIC 3052, Australia. 'School of Women's and Infants' Health, University of Western Australia, Crawley, WA, Australia. ${ }^{7}$ Centre for Population Health Research, Deakin University, Burwood, VIC 3125, Australia.

Received: 17 August 2015 Accepted: 9 December 2015

Published online: 16 December 2015

\section{References}

1. Amir LH, Forster DA, Lumley J, McLachlan H. A descriptive study of mastitis in Australian breastfeeding women: incidence and determinants. BMC Public Health. 2007;7:62.

2. Kinlay JR, O'Connell DL, Kinlay S. Incidence of mastitis in breastfeeding women during the six months after delivery: a prospective cohort study. Med J Aust. 1998;169(6):310-2.

3. Vogel A, Hutchison BL, Mitchell EA. Mastitis in the first year postpartum. Birth. 1999;26(4):218-25. 
4. Amir LH, Lumley J. Women's experience of mastitis: 'I have never felt worse'. Aust Fam Physician. 2006;35(9):745-7.

5. Wambach KA. Lactation mastitis: a descriptive study of the experience. J Hum Lact. 2003;19(1):24-34.

6. Fetherston C. Management of lactation mastitis in a Western Australian cohort. Breastfeed Rev. 1997;5(2):13-9.

7. Amir LH, Trupin S, Kvist $\sqcup$. Diagnosis and treatment of mastitis in breastfeeding women. J Hum Lact. 2014:30(1):10-3.

8. Feijen-de Jong El, Baarveld F, Jansen DE, Ursum J, Reijneveld SA, Schellevis FG. Do pregnant women contact their general practitioner? A register-based comparison of healthcare utilisation of pregnant and non-pregnant women in general practice. BMC Fam Pract. 2013;14:10.

9. Amir LH, The Academy of Breastfeeding Medicine Protocol Committee. ABM Clinical Protocol \# 4: Mastitis, Revision, March 2014. Breastfeed Med. 2014;9(5):239-43.

10. Amir LH, Forster D, McLachlan H, Lumley J. Incidence of breast abscess in lactating women: report from an Australian cohort. BJOG. 2004;111(12): $1378-81$.

11. World Health Organization. Mastitis: causes and management. Geneva: WHO/FCH/ CAH/00.13; 2000.

12. Fetherston C. Risk factors for lactation mastitis. J Hum Lact. 1998;14(2):101-9.

13. Foxman B, D'Arcy H, Gillespie B, Bobo JK, Schwartz K. Lactation mastitis: occurrence and medical management among 946 breastfeeding women in the United States. Am J Epidemiol. 2002;155(2):103-14.

14. Kinlay JR, O'Connell DL, Kinlay S. Risk factors for mastitis in breastfeeding women: results of a prospective cohort study. Aust N Z J Public Health. 2001;25(2):115-20.

15. Vogel A, Hutchison BL, Mitchell EA. Factors associated with the duration of breastfeeding. Acta Paediatr. 1999;88(12):1320-6.

16. Scott JA, Robertson M, Fitzpatrick J, Knight C, Mulholland S. Occurrence of lactational mastitis and medical management: a prospective cohort study in Glasgow. Int Breastfeed J. 2008;3:21.

17. Nicholson W, Yuen HP. A study of breast feeding rates at a large Australian obstetric hospital. Aust N Z J Obstet Gynaecol. 1995;35(4):393-7.

18. Waldenstrom U, Nilsson CA. No effect of birth centre care on either duration or experience of breast feeding, but more complications: findings from a randomised controlled trial. Midwifery. 1994;10(1):8-17.

19. Johns HM, Forster DA, Amir LH, Moorhead AM, McEgan KM, McLachlan HL. Infant feeding practices in the first 24-48 $\mathrm{h}$ of life in healthy term infants. Acta Paediatr. 2013;102(7):e315-20.

20. Forster DA, Johns HM, McLachlan HL, Moorhead AM, McEgan KM, Amir LH. Feeding infants directly at the breast during the postpartum hospital stay is associated with increased breastfeeding at 6 months postpartum: a prospective cohort study. BMJ Open. 2015;5(5):e007512.

21. Mediano P, Fernandez L, Rodriguez JM, Marin M. Case-control study of risk factors for infectious mastitis in Spanish breastfeeding women. BMC Pregnancy Childbirth. 2014;14:195.

22. Kvist $L$, Larsson BW, Hall-Lord ML, Steen A, Schalen C. The role of bacteria in lactational mastitis and some considerations of the use of antibiotic treatment. Int Breastfeed J. 2008;3:6.

23. Osterman KL, Rahm VA. Lactation mastitis: bacterial cultivation of breast milk, symptoms, treatment and outcome. J Hum Lact. 2000;16(4):297-302.

24. Amir LH, Garland SM, Lumley J. A case-control study of mastitis: nasal carriage of Staphylococcus aureus. BMC Fam Pract. 2006;7:57.

25. Delgado $S$, Arroyo R, Jiménez E, Marín M, del Campo R, Fernández L, et al. Staphylococcus epidermidis strains isolated from breast milk of women suffering infectious mastitis: potential virulence traits and resistance to antibiotics. BMC Microbiol. 2009;8:82.

26. Witt A, Mason MJ, Burgess K, Flocke S, Zyzanski S. A case control study of bacterial species and colony count in milk of breastfeeding women with chronic pain. Breastfeed Med. 2014;9(1):29-34.

27. Amir LH, Cullinane M, Garland SM, Tabrizi SN, Donath SM, Bennett CM, et al. The role of micro-organisms (Staphylococcus aureus and Candida albicans) in the pathogenesis of breast pain and infection in lactating women: study protocol. BMC Pregnancy Childbirth. 2011;11:54.

28. Amir LH, Donath SM, Garland SM, Tabrizi SN, Bennett CM, Cullinane M, et al. Does Candida and/or Staphylococcus play a role in nipple and breast pain in lactation? A cohort study in Melbourne. Australia BMJ Open. 2013;3, e002351.

29. Clemons SN, Amir LH. Breastfeeding women's experience of expressing: a descriptive study. J Hum Lact. 2010;26(3):258-65.
30. Buck ML, Amir LH, Cullinane M, Donath SM, CASTLE study team. Nipple pain, damage and vasospasm in the first eight weeks postpartum. Breastfeed Med. 2014;9(2):56-62.

31. Buck ML, Amir LH, Donath SM. Topical treatments used by breastfeeding women to treat sore and damaged nipples. Clinical Lactation. 2015;6(1):16-23.

32. Forster $\mathrm{D}, \mathrm{McL}$ achlan $\mathrm{H}$, Lumley J, Beanland C, Waldenström U, Harris H, et al. Study protocol. ABFAB. Attachment to the breast and family attitudes to breastfeeding. The effect of breastfeeding education in the middle of pregnancy on the initiation and duration of breastfeeding: a randomised controlled trial [ISRCTN21556494]. BMC Pregnancy Childbirth. 2003;3:5.

33. Carlin JB, Wolfe R, Coffey C, Patton GC. Analysis of binary outcomes in longitudinal studies using weighted estimating equations and discrete-time survival methods: prevalence and incidence of smoking in an adolescent cohort. Stat Med. 1999;18(19):2655-79.

34. Stata 13. College Station, Texas, USA: StataCorp LP; 2013.

35. Jonsson S, Pulkkinen MO. Mastitis today: incidence, prevention and treatment. Ann Chir Gynaecol Suppl. 1994;83:84-7.

36. Fetherston C. Characteristics of lactation mastitis in a Western Australian cohort. Breastfeed Rev. 1997;5(2):5-11.

37. Thompson JF, Roberts CL, Currie M, Ellwood DA. Prevalence and persistence of health problems after childbirth: associations with parity and method of birth. Birth. 2002;29(2):83-94.

38. Foxman B, Schwartz K, Looman SJ. Breastfeeding practices and lactation mastitis. Soc Sci Med. 1994;38(5):755-61.

39. Johns HM, Amir LH, McLachlan HL, Forster DA. Breast pump use amongst mothers of healthy term infants in Melbourne, Australia: a prospective cohort study. Midwifery. 2015. [Epub ahead of print].

40. von Eiff C, Becker K, Machka K, Stammer H, Peters G. Nasal carriage as a source of Staphylococcus aureus bacteremia. N Engl J Med. 2001;344(1):11-6.

41. Kawada M, Okuzumi K, Hitomi S, Sugishita C. Transmission of Staphylococcus aureus between healthy, lactating mothers and their infants by breastfeeding. J Hum Lact. 2003;19(4):411-7.

42. Ingman W, Glynn DJ, Hutchinson MR. Mouse models of mastitis - how physiological are they? Int Breastfeed J. 2015;10:12.

43. Amir LH. Managing common breastfeeding problems in the community. BMJ. 2014;348:g2954.

\section{Submit your next manuscript to BioMed Central and we will help you at every step:}

- We accept pre-submission inquiries

- Our selector tool helps you to find the most relevant journal

- We provide round the clock customer support

- Convenient online submission

- Thorough peer review

- Inclusion in PubMed and all major indexing services

- Maximum visibility for your research

Submit your manuscript at www.biomedcentral.com/submit

) Biomed Central 